\title{
Study of the Structure and Electronic Properties of the ZnO Monolayer: Density Functional Theory
}

\author{
Muhammad Fadlan Raihan ${ }^{1, a}$, Triati Dewi Kencana Wungu, ${ }^{1,3, b}$ and Brian \\ Yuliarto $^{2,3}$
}

${ }^{1}$ Department of Physics, Faculty of Mathematics and Natural Sciences, Institut Teknologi Bandung, Jl. Ganesa No. 10 Bandung 40132, Indonesia

${ }^{2}$ Department of Engineering Physics, Faculty of Industrial Technology, Institut Teknologi Bandung, Jl. Ganesa No. 10 Bandung 40132, Indonesia

${ }^{3}$ Research Center for Nanosciences and Nanotechnology, Institut Teknologi Bandung, J. Ganesa No. 10 Bandung 40132, Indonesia

afraihan1710@gmail.com, ${ }^{b}$ triati@fi.itb.ac.id

\begin{abstract}
ZnO}$ has received considerable attention since it has promising applications in electronic devices. Although many studies have explored the potential of $\mathrm{ZnO}$ as a promising material, the precise role of geometric in $\mathrm{ZnO}$ remains unclear. This study deals with the electronic structure of the $\mathrm{ZnO}$ Monolayer using Density Functional Theory (DFT). The DFT was used to investigate the band structure and density of states of the $\mathrm{ZnO}$ Monolayer. It is observed that the structural change of $\mathrm{ZnO}$ from bulk to monolayer increases the bandgap by $1.84 \mathrm{eV}$ without changes its natural characteristic. Moreover, This study provides information about the properties of the $\mathrm{ZnO}$ Monolayer and its potential in electronic and magnetic devices application.
\end{abstract}

Keywords: DFT, Monolayer ZnO, Band-gap

\section{Introduction}

Semiconducting metal oxides have been extensively studied as functional materials for wide applications relating to sustainable development, such as energy conversion/storage [1], environmental remediation [2], and high-performance electronics [3]. Based on simplicity in synthesis, nontoxicity, and relatively low-cost production, $\mathrm{ZnO}$ becomes one of the semiconducting metal oxides that have been used in many applied technologies [4]. Due to the fact that $\mathrm{ZnO}$ has its uniques properties, huge demand in manufacturing $\mathrm{ZnO}$ provides new opportunities for tuning the nano and mesoscale structures of $\mathrm{ZnO}$ to extend its applications [5].

In recent years, $\mathrm{ZnO}$ nanostructures such as nanowires, nanobelts, and nanorings have been synthesized for electronic devices such as field-effect transistors [6], supercapacitors [7], and gas sensors [8]. Moreover, several studies have also found that the $\mathrm{Zn}$ atoms can induce ferromagnetic properties on thin films of $\mathrm{ZnO}$ nanowires without doping transition metal atoms, which commonly are harmful to humans. Thus, $\mathrm{ZnO}$ semiconductor material can be used in the biomedical field due to it does not have harmful toxins [9].

The potential of $\mathrm{ZnO}$ as a promising material still needs to be explored to enhance its performance. One of the techniques that can be used is by modifying the morphology of $\mathrm{ZnO}$ into a monolayer structure. However, modifying the morphology in an actual experiment is 
unpredictable and requires many trials and errors. Therefore, we need a simulation approach to predict the resulting morphology in the modification of $\mathrm{ZnO}$. The morphological modification of various materials, especially $\mathrm{ZnO}$, can be predicted using the Density Functional Theory (DFT) simulation method because the obtained calculations are close to the experimental results [10]. In this study, the ZnO Monolayer will be simulated using DFT to analyze the geometry and electronics properties of the material.

\section{Computational Details}

The monolayer structure of $\mathrm{ZnO}$ was obtained by cutting the bulk structure of $\mathrm{ZnO}$ at $\left[\begin{array}{lll}0 & 0 & 1\end{array}\right]$ orientation (Figure 1). This treatment is consistent with the structure of the $\mathrm{ZnO}$ Monolayer, which was experimentally synthesized by Young and Lai [11]. We constructed a periodic (4 x 4) supercell of $\mathrm{ZnO}$ Monolayer with a $15 \AA$ vacuum space in all models to avoid interactions between adjacent layers.

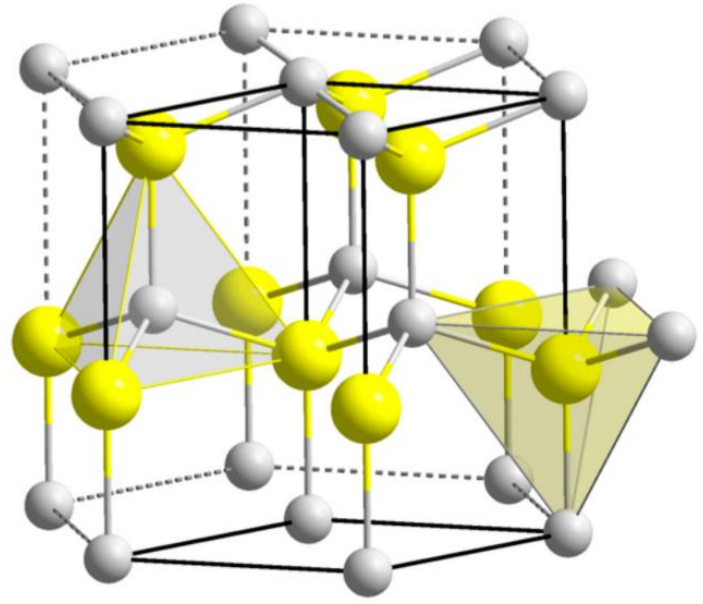

Figure 1. Bulk structure of $\mathrm{ZnO}$; $\mathrm{Zn}$ (yellow), and O (grey) [12]

The electronic properties and geometrical structure of the $\mathrm{ZnO}$ Monolayer were investigated by the Density Functional Theory (DFT) method. All calculations were performed using PerdewBurke-Ernzerhof (PBE) exchange-correlation functional implemented in the Vienna Ab initio Simulation Package (VASP) [13]. For the plane-wave basis set, an energy cutoff of $490 \mathrm{eV}$ was used. The maximal force of $0.01 \mathrm{eV} / \AA$ was used as the convergence criterion for ionic relaxations, and $10^{-5}$ was also used for convergence tolerances. The Brillouin Zone was sampled Monkhorst-Pack mesh [14] with $5 \times 5 \times 1$ k-points in the geometry optimization and $20 \times 20 \times 1 \mathrm{k}$-points arranged in the electronic structure [14]. The fully relaxed geometry was performed to obtain the optimized structure. The electronic structure is analyzed by calculating the band structure and the Density of State (DOS).

\section{Results and Discussion}

After optimizing the geometry, the layer of $\mathrm{ZnO}$ Monolayer changed from the initial structure with a rippled surface into a honeycomb-like structure similar to graphene (Figure 2). Interestingly, 
the calculated $\mathrm{Zn}-\mathrm{O}$ bond length is $1.876 \AA$ which is shorter than the bulk structure [15]. This result is related to the fact that the atoms in the monolayer structure have a lower coordination number than the bulk structure. Hence, the atoms in the $\mathrm{ZnO}$ Monolayer strengthen the bonds between the $\mathrm{Zn}-\mathrm{O}$ atoms themselves and shorten the bond length.

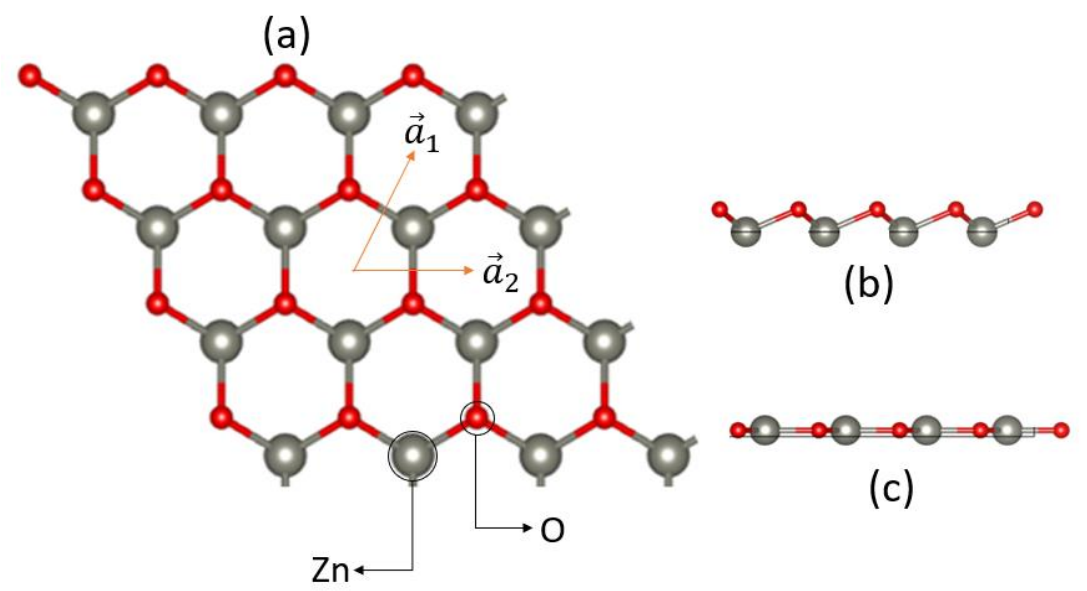

Figure 2. Monolayer Structure of ZnO; (a) Top View; (b) Side View before optimization; (c) Side View after optimization.

To determine the electronic properties of the $\mathrm{ZnO}$ Monolayer, it is necessary to calculate the band structure and Density of State (DOS) of the material; the result of the calculation can be seen in Figures 3 and 4.

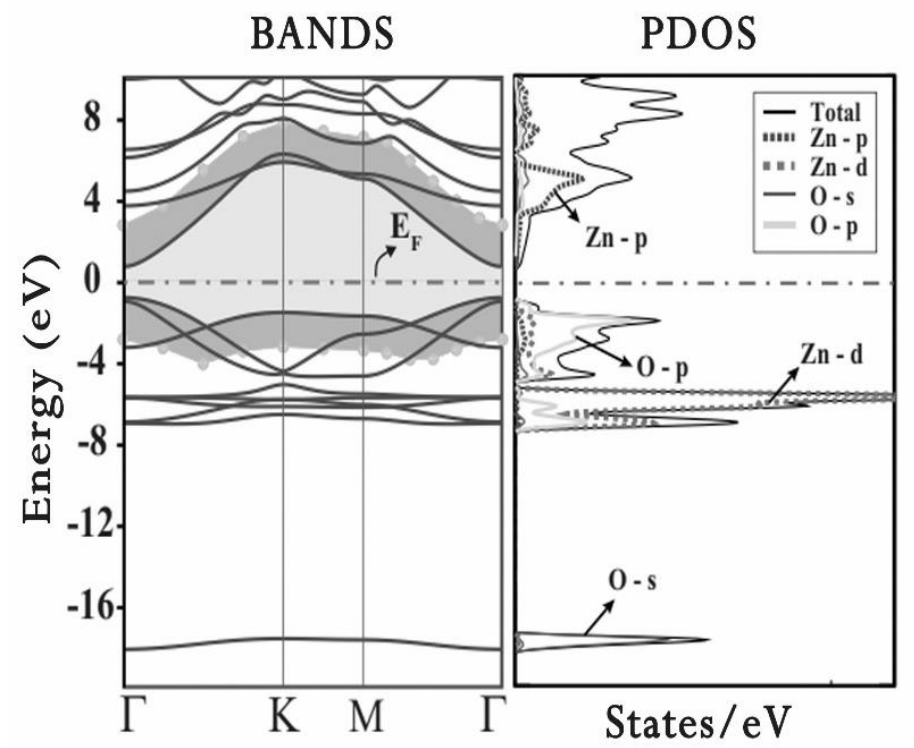

Figure 3. Band Structure and Partial Density of States (PDOS) 
The PDOS in Figure 3 shows that the high density in the conduction band is dominated by O-2p atoms while the valence band is dominated by $\mathrm{Zn}-3 \mathrm{~d}$ atoms, which similar to the bulk structure. This result indicates that the $\mathrm{Zn}$ atom transfers its charge to the $\mathrm{O}$ atom, forming a $\mathrm{Zn}-\mathrm{O}$ bond with ionic interaction.

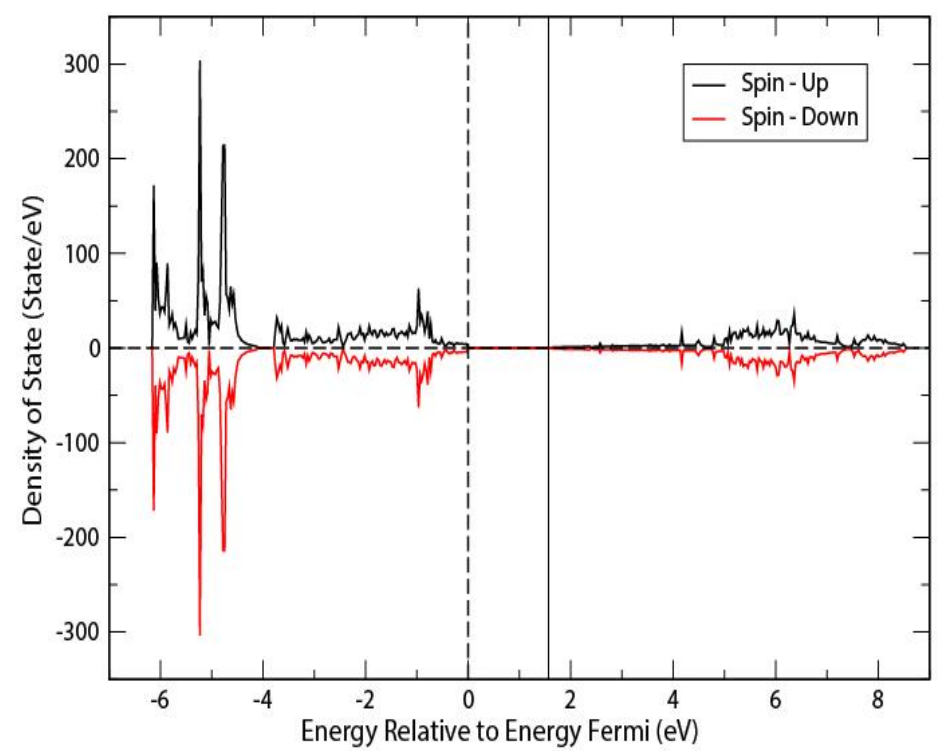

Figure 4. Total Density of States (TDOS)

As shown in Figure 4, the electronic state densities are symmetrical for both spin-up and spindown. The symmetrical electron density causes the magnetic moment of this material to be zero, indicating that $\mathrm{ZnO}$ is a non-magnetic semiconductor material.

Table 1. The Energy Gap of $\mathrm{ZnO}$ Materials with Various Nanostructures

\begin{tabular}{lll}
\hline Material & Structure & GGA \\
\hline & Wurtzite & $0.75 \mathrm{eV}[16]$ \\
& Zincblende & $0.65 \mathrm{eV}[17]$ \\
\multirow{3}{*}{ ZnO } & Nanowire & $0.71 \mathrm{eV}[18]$ \\
& & $0.91 \mathrm{eV}[19]$ \\
& Nanoribbon & $1.76 \mathrm{eV}[20]$ \\
& Monolayer & $1.30 \mathrm{eV}[21]$ \\
& & $1.84 \mathrm{eV}$ (This study) \\
\hline
\end{tabular}

In addition, The comparison of the Generalized Gradient Approximation (GGA) bandgap of ZnO semiconductors with various nanostructures is presented in Table 1. The Monolayer $\mathrm{ZnO}$ has a direct bandgap of $1.84 \mathrm{eV}$ (Figure 4). This result was higher compared to the other reported nanostructure. The fact also shows that the $\mathrm{ZnO}$ Monolayer has a larger bandgap compared to nanowire and nanoribbon structures. The bandgap is the forbidden region to be occupied by electrons. A larger forbidden region signifies a greater restriction on the movement of electrons [22]. Moreover, the large energy bandgap in the UV region and fast recombination rate of 
electron-hole pairs retards its application as a photocatalyst under direct sunlight [23]. These results indicate that Monolayer $\mathrm{ZnO}$ can be applied in nanoscale photoelectronic devices, especially for UV photon emitters and detectors.

\section{Conclusions}

The structure and electronic properties of the $\mathrm{ZnO}$ Monolayer have been investigated using Density Functional Theory (DFT) calculations. The calculation results show that the modification of the $\mathrm{ZnO}$ Monolayer structure can provide an alternative to increasing the bandgap without changing the characteristics of $\mathrm{ZnO}$ as a semiconductor with a direct bandgap. These results indicate that Monolayer ZnO can be applied in electronic devices such as UV photon emitters and detectors. The DOS analysis also shows that the $\mathrm{ZnO}$ Monolayer has a significant charge transfer from the Zinc $(\mathrm{Zn})$ atom to the nearest Oxygen $(\mathrm{O})$ atom.

\section{ACKNOWLEDGEMENTS}

The authors acknowledge the Research Center for Nanosciences and Nanotechnology (PPNN), Institut Teknologi Bandung, Indonesia, for providing technical support and calculation facilities. This study was supported by a grant from The National Research and Innovation Agency Indonesia (RISTEK-BRIN) 2021.

\section{References}

[1] H. Wang et al., Jun. 2015, Bifunctional non-noble metal oxide nanoparticle electrocatalysts through lithium-induced conversion for overall water splitting, Nat. Commun, volume 6, no. 1, page 1-8.

[2] Z. Zhang, J. Liu, J. Gu, L. Su and L. Cheng, Jul. 18, 2014, An overview of metal oxide materials as electrocatalysts and supports for polymer electrolyte fuel cells, Energy and Environmental Science, volume 7, no. 8, Royal Society of Chemistry, page 2535-2558.

[3] S. Park, C. H. Kim, W. J. Lee, S. Sung, and M. H. Yoon, Apr. 01, 2017, Sol-gel metal oxide dielectrics for all-solution-processed electronics, Materials Science and Engineering R: Reports, volume 114. Elsevier Ltd, page 1-22.

[4] N. A. Abdullah, Z. Khusaimi, and M. Rusop, 2013, A review on zinc oxide nanostructures: Doping and gas sensing, in Advanced Materials Research, volume 667, page 329-332.

[5] S. Y. Wakhare and M. D. Deshpande, Oct. 2019, The electronic and optical properties of monovalent atom-doped ZnO monolayers: the density functional theory, Bull. Mater. Sci., volume 42, no.5, page 206.

[6] Y. Li et al., Apr. 2017, Enhanced Performance in Al-Doped ZnO Based Transparent Flexible Transparent Thin-Film Transistors Due to Oxygen Vacancy in ZnO Film with ZnAl-O Interfaces Fabricated by Atomic Layer Deposition, ACS Appl. Mater. Interfaces, volume 9, no. 13, page 11711-11720.

[7] D. Cai et al., Sep. 2014, High-performance supercapacitor electrode based on the unique ZnO@Co3O4 core/shell heterostructures on nickel foam, ACS Appl. Mater. Interfaces, volume 6, no. 18, page 15905-15912.

[8] Y.-F. Sun et al., Feb. 2012, Metal Oxide Nanostructures and Their Gas Sensing 
Properties: A Review, Sensors, volume12, no.3, page 2610-2631.

[9] Q. Wang, Q. Sun, G. Chen, Y. Kawazoe, and P. Jena, May 2008, Vacancy-induced magnetism in ZnO thin films and nanowires, Phys. Rev. B - Condens. Matter Mater. Phys., volume 77, no. 20, page 205411.

[10] C. Szakacs, E. Merschrod S., and K. Poduska, May 2013, Structural Features That Stabilize ZnO Clusters: An Electronic Structure Approach, Computation, volume 1, no. 1, page 16-26.

[11] S. J. Young and L. T. Lai, Dec. 2015, Field emission properties of ZnO nanosheets grown on a Si substrate, Microelectron. Eng., volume 148, page 40-43.

[12] M. A. Borysiewicz, Oct. 2019, ZnO as a functional material, a review, Crystals, volume 9, no. 10, page 505 .

[13] G. Kresse and J. Furthmüller, Oct. 1996, Efficient iterative schemes for ab initio totalenergy calculations using a plane-wave basis set, Phys. Rev. B - Condens. Matter Mater. Phys., volume 54, no. 16, page 11169-11186.

[14] H. J. Monkhorst and J. D. Pack, Jun. 1976, Special points for Brillouin-zone integrations, Phys. Rev. B, volume 13, no. 12, page 5188-5192.

[15] D. Q. Fang, A. L. Rosa, R. Q. Zhang, and T. Frauenheim, Apr. 2010, Theoretical exploration of the structural, electronic, and magnetic properties of $\mathrm{ZnO}$ nanotubes with vacancies, antisites, and nitrogen substitutional defects, J. Phys. Chem. C, volume 114, no. 13, page 5760-5766.

[16] M. Usuda, N. Hamada, T. Kotani, and M. Van Schilfgaarde, Sep. 2002, All-electron (formula presented) calculation based on the LAPW method: Application to wurtzite ZnO, Phys. Rev. B - Condens. Matter Mater. Phys., volume 66, no. 12, page 1-8.

[17] M. Oshikiri and F. Aryasetiawan, Oct. 1999, Band gaps and quasiparticle energy calculations on $\mathrm{ZnO}, \mathrm{ZnS}$, and $\mathrm{ZnSe}$ in the zinc-blende structure by the GW approximation, Phys. Rev. B - Condens. Matter Mater. Phys., volume 60, no. 15, page 10754-10757.

[18] M. Srivastava and A. Srivastava, Aug. 2019, Electron transport in CO2 adsorbed ZnO nanowire: DFT study, Chem. Phys. Lett., volume 729, page 17-23.

[19] F. C. Zhang, Z. Y. Zhang, W. H. Zhang, J. F. Yan, and J. N. Yong, Jun. 2009, Firstprinciples study of the electronic and optical properties of ZnO nanowires, Chinese Phys. B, volume 18, no. 6, page 2508-2513.

[20] A. De Sarkar and R. Ahuja, Nov. 2014, Electronic charge transport through ZnO nanoribbons, J. Phys. Chem. Solids, volume 75, no. 11, page 1223-1228.

[21] P. Singh, D. K. K. Randhawa, Tarun, B. C. Choudhary, G. K. Walia, and N. Kaur, Jan. 2020, First-principles investigation on armchair zinc oxide nanoribbons as uric acid sensors, J. Mol. Model, volume 26, no. 1, page 1-11.

[22] A. Samavati et al., Jan. 2021, Influence of ZnO nanostructure configuration on tailoring the optical bandgap: Theory and experiment, Mater. Sci. Eng. B Solid-State Mater. Adv. Technol., volume 263, page 114811.

[23] M. S. Nadeem, T. Munawar, F. Mukhtar, M. Naveed ur Rahman, M. Riaz, and F. Iqbal, 
Apr. 2021, Enhancement in the photocatalytic and antimicrobial properties of ZnO nanoparticles by structural variations and energy bandgap tuning through $\mathrm{Fe}$ and $\mathrm{Co}$ codoping, Ceram. Int., volume 47, no. 8, page 11109-11121. 